\title{
Panorama de la construcción de vivienda sostenible e incluyente en Colombia
}

\section{Overview of sustainable and inclusive house building in Colombia}

\author{
Diego Gómez Calderón ${ }^{1}$ \\ Carlos Duque Gallego ${ }^{2}$ \\ Fecha de recepción: 20/11/2020 - Fecha de aceptación: 10/12/2020
}

DOI: https://doi.org/10.22490/26655489.4219

\section{Resumen}

El desarrollo sostenible expresa el vínculo entre el desarrollo económico y social y sus efectos sobre el medio ambiente. Lo anterior no es en sí mismo una problemática nueva; lo novedoso es la magnitud y extensión alcanzada en los últimos años. A este desafío se le suma la necesidad de generar espacios públicos y habitacionales incluyentes con la población con discapacidad física. Estos dos elementos establecen un reto para el sector de la vivienda, ya que en esta dinámica se concretan los impactos frente a la mitigación de los fenómenos descritos. El Estado, desde esta perspectiva, adquiere un rol protagónico toda vez que promueve y regula el acceso a la vivienda, siendo el principal garante de acceso a vivienda de población vulnerable. Definir un panorama general sobre la materia es el objeto central del presente documento.

Palabras clave: medio ambiente, población con movilidad reducida, energía solar y viviendas sostenibles.

\footnotetext{
1 Doctorando en Economía y Gobierno de la Universidad Internacional Menéndez Pelayo y el Instituto Universitario de Investigación Ortega y Gasset (España). Magister en Economía de la Universidad del Valle y Economista de la Universidad Libre. Profesor y miembro del grupo de investigación Política, Derecho y Territorio - PODET - de la Universidad Nacional Abierta y a Distancia - UNAD -, Cali, Colombia, correo electrónico: diegoj.gomez@unad.edu.co ORCID: 0000-0002-2619-5673
}

2 Magíster en Administración de Empresas de la Universidad del Valle. Especialista en Finanzas Universidad ICESI y Administrador de Empresas Universidad Santiago de Cali. Profesor e investigador de la Universidad Antonio Nariño, Cali, Colombia, correo electrónico: carlosduque@uan.edu.co ORCID: 0000-0003-4950-0252 


\section{Abstract}

Sustainable development expresses the link between economic and social development and its effects on the environment. The above is not in itself a new problem, the novelty is the magnitude and extent achieved in recent years. To this challenge is added the need to generate inclusive public and residential spaces with the population with physical disabilities. These two elements establish a challenge for the housing sector, since this dynamic specifies the impacts against the mitigation of the described phenomena. The State, from this perspective, acquires a leading role since it promotes and regulates access to housing, being the main guarantor of access to housing for vulnerable populations. Defining a general overview of the subject is the main purpose of this document.

Keywords: environment, population with reduced mobility, solar energy and sustainable housing

\section{Introducción}

La sociedad moderna tiene como uno de sus rasgos distintivos, entre los muchos que pueden definirse, al confort como una variable que establece diferenciadores sociales y de calidad de vida. Entre los diferentes contextos donde se desarrolla la vida moderna con criterios de confort, la vivienda, es decir, el espacio habitacional, es uno de dichos dispositivos que ha sido sujeto de múltiples cambios, todos orientados a brindar mayor confort y seguridad.

La presión generada por el incremento estructural del tamaño poblacional a nivel mundial, y principalmente en países en vía de desarrollo durante el siglo $X X$, ha generado todo un engranaje de política pública que permite el acceso a la vivienda como garantía de bienestar social. El acceso ha sido la fuente de las múltiples políticas que en el caso colombiano han motivado los diferentes escenarios para potenciar desde el Estado la construcción de vivienda, así como la regulación desde el ordenamiento territorial, condiciones técnicas y de materiales, etc., que el Estado desarrolla para las iniciativas privadas sobre la materia. 
Los inmensos desafíos que implica el cambio climático y la contaminación al medio ambiente; así como la generación de espacios públicos y/o habitacionales de fácil acceso a población en condiciones de discapacidad física, han impactado el sector de la vivienda y al Estado como ente que dinamiza las condiciones del sector y posibilita el acceso a la vivienda de población vulnerable. Desde esta perspectiva, el presente documento construye un panorama sobre la dinámica de construcción de vivienda sostenible e incluyente en Colombia, lo cual permite definir la necesidad de generar política púbica sobre la construcción de vivienda con rasgos diferenciadores sobre la autosostenibilidad ambiental y la arquitectura incluyente. Integrando estos tres ejes se puede responder a tres de las problemáticas actuales de los países en vía de desarrollo, como es el caso de Colombia.

Desde esta perspectiva el siguiente documento se compone de la presente introducción; en el apartado uno se definen los elementos conceptuales básicos de la sostenibilidad y de la inclusión, en el marco de los objetivos de desarrollo sostenible. En el apartado dos se realiza una caracterización de la dinámica del sector de construcciones en Colombia, y en el tres se presentan algunas estadísticas sobre la población con movilidad reducida en Colombia y América Latina. Por último, se presentan unas ideas finales a manera de conclusión.

\section{Objetivos de Desarrollo Sostenible}

Los organismos multilaterales entre ellos las Naciones Unidas, en el año 2015 de forma unánime dieron via libre a 17 objetivos de cobertura mundial que se denominaron los Objetivos de Desarrollo Sostenible (de ahora en adelante ODS), como una acción conjunto mundial para enfrentar los retos planteados por el incremento acelerado de la pobreza, y garantizar la protección del planeta en general. Los ODS fueron estructurados como parte de la agenda 2030 que es el plazo para la realización de dicha propuesta.

En el ámbito académico se están logrando algunos avances, como son los lineamientos en investigación de parte del sector educativo de la educación superior, en donde los actores (universidades) están exigiendo a los investigadores que los proyectos se articulen a los ODS y de esta manera se dinamiza el proceso en la relación universidad-sector productivo. En el año 
2019 se realizó la cumbre sobre los ODS en las Naciones Unidas; en este evento los representantes de las diferentes naciones, dados los plazos perentorios de 10 años para el cumplimento de los objetivos propuestos, se comprometieron con la asignación de recursos para la financiación de los ODS. En esta línea, la Secretaría General de la ONU, convocó a toda la comunidad mundial en torno a este propósito de interés global en tres niveles fundamentales:

a. Enfoque mundial en el logro de mayor liderazgo para la orientación de los recursos.

b. Nivel local que implique los alineamientos de las políticas públicas, las políticas institucionales del sector educativo superior al logro de los ODS y la concientización de la sociedad civil en general a través de instituciones como los medios de comunicación, los sindicatos, los círculos académicos.

c. El sector privado para que todos en conjunto se conviertan en multiplicadores en las transformaciones que se necesitan para la sostenibilidad y la vida en el planeta.

\subsection{Sostenibilidad ambiental: el gran desafío del siglo XXI}

El desarrollo sostenible expresa el vínculo entre el desarrollo económico y social y sus efectos sobre el medio ambiente (natural). Lo anterior no es en sí mismo una problemática nueva; lo novedoso es la magnitud y extensión alcanzada en los últimos años, lo cual ha conducido a una estimación sobre sus consecuencias futuras, incluida la capacidad de supervivencia de la especie humana. Por esta razón, diferentes agencias internacionales y locales de todo orden y naturaleza, así como gobiernos nacionales, regionales y locales, han abierto un espacio importante para la agenda ambiental, la cual ha ganado importancia, en términos de recursos financieros destinados y acciones de divulgación. 
Global Footprint Network (2019), una organización sin fines de lucro de carácter ambiental desarrolla la encuesta anual sobre el uso que hace la humanidad de los recursos de la Tierra. A partir de esta encuesta, la organización diseñó un indicador llamado "Earth Overshoot Day", el cual expresa el punto en el año calendario en que la explotación de los recursos biológicos de la humanidad excede la capacidad del planeta de regenerar recursos para ese año. Esto significa que el resto del año, todos los recursos consumidos representan un sobregiro ecológico y, por lo tanto, son insostenibles.

Figura 1. Earth Overshoot Day (sobregiro ecológico)

1980 - 2018

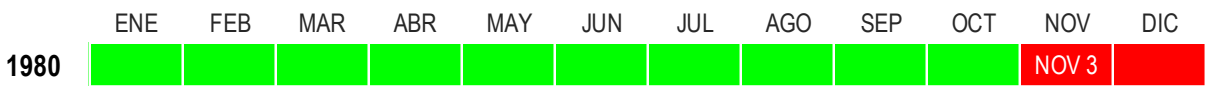

2000

OCT 4

2018

AGO 1

Fuente: Construcción propia con datos de Global Footprint Network National Footprint Accounts 2019.

En la Figura 1 se muestran los resultados de este indicador para 1980,2000 y 2018. Por ejemplo, en 1980 la población mundial consumió los recursos disponibles para ese año el 3 de noviembre, mientras que en 2018 , ese consumo se alcanzó en agosto 1. Esa es una forma simple, como El Earth Overshoot Day evidencia la enorme problemática de la sostenibilidad del medio ambiente, ya que el ritmo de sobregiro ecológico es mayor y cada vez alcanza un mayor ritmo de crecimiento.

Según la misma organización, el país que más consume recursos es Estados Unidos, al punto que, si todos los ciudadanos del mundo vivieran como un ciudadano estadounidense promedio, se requerirían cinco planetas para satisfacer la demanda de recursos. Australia ocupa el segundo lugar, seguido por Corea del Sur, Rusia y Alemania (Ver Figura 2). 
Figura 2. Necesidad de planetas para vivir según principales países.

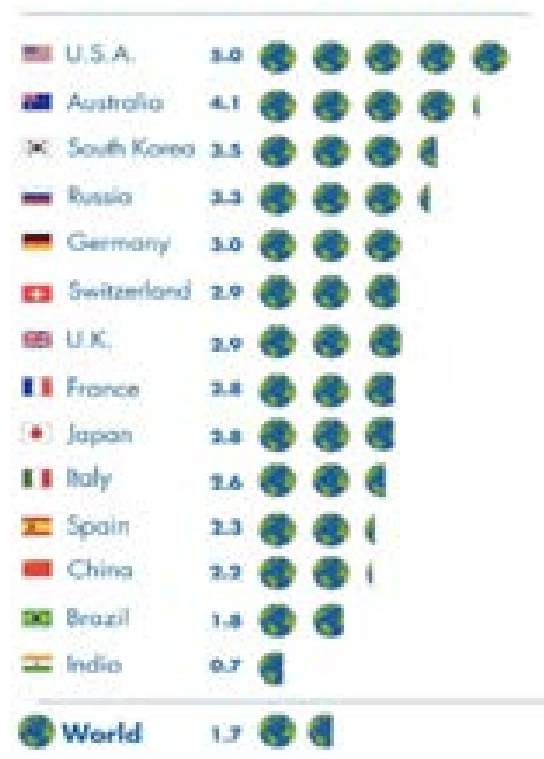

Fuente: Tomado de Global Footprint Network National Footprint Accounts 2018.

La definición más citada de desarrollo sostenible es de la Comisión Brudtland (WCED 1987, p. 8) "progreso que satisface las necesidades del presente sin comprometer la capacidad de las generaciones futuras para satisfacer sus propias necesidades". Esta definición resalta el legado que deja una generación a la siguiente en términos de bienestar, por ende, el desarrollo sostenible se entiende como el constante mejoramiento del bienestar humano a través del tiempo.

Desde esta perspectiva, Wackernagel et al. (1999), proponen el indicador de Huella Ecológica, el cual representa "el capital natural que demanda una economía y se determina calculando el consumo de recursos y los requerimientos de asimilación de residuos de una población humana definida en términos de área de tierra productiva correspondiente" (Arias, 2006 , p. 202). En este sentido, la huella ecológica mide la carga impuesta por una población sobre la naturaleza en términos del área de tierra requerida para mantener la actividad económica, así que el común denominador hace referencia a las hectáreas de tierra ecológicamente productiva. 
En el mismo sentido, se desprende el concepto de biocapacidad, definida como "...la capacidad de un área biológicamente productiva dada, para generar un suministro continuo de recursos renovables y para absorber sus desechos" (Global Footprint Network, 2019, tomado de http://data. footprintnetwork.org/\#/country), por lo tanto, la insostenibilidad de un territorio se produce si la huella ecológica del área supera su biocapacidad.

La huella ecológica per cápita mundial en 2016 fue 2,75 ha y la biocapacidad per cápita fue 1,63 ha ese mismo año; es decir, el mundo sostiene un déficit ecológico por cada individuo de $-1,12$ ha. En particular para Colombia, la huella ecológica per cápita en 2016 fue de 2,05 ha y su biocapacidad fue 3,64, lo cual indica que el país, a diferencia del mundo, aún tiene un superávit ecológico, que asciende a 1,59 ha/per cápita (Ver Figura 3).

Figura 3. Huella Ecológica vs Biocapacidad ha. Por persona* para Colombia, 1961-2014

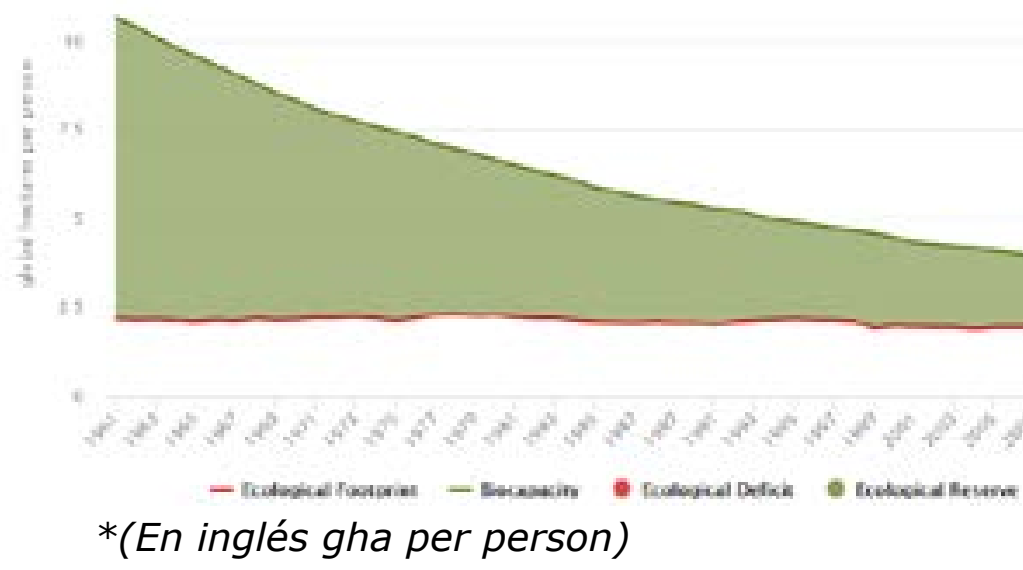

Fuente: Global Footprint Network National Footprint Accounts 2019. 
Aunque el país genera un superávit ecológico, se puede observar cómo esta dinámica superavitaria se viene reduciendo en los últimos años, lo cual obliga a mirar el tema de la energía y los combustibles como causas estructurales de dicho deterioro ambiental. Según la UPME (2017), el consumo de energía se concentra en los sectores transporte $(40,1 \%)$, industrial $(29,4$ $\%)$, residencial $(16,7 \%)$ y sector terciario (5\%). El $70 \%$ de la energía eléctrica que se consume en los hogares está relacionada con el uso del aire acondicionado, ventilación y electrodomésticos.

Uno de los principales efectos del deterioro ambiental en el país, está relacionado con un riesgo potencial de suministro de agua a través del descontrol hídrico y pluviométrico, incrementando de paso el mayor riesgo de sequías e inundaciones (WWF-Colombia, 2017). La UPME (2018) indicó que el $70 \%$ de la energía utilizada en Colombia en 2018 provino de hidroeléctricas, lo que demuestra la importancia de conservar las cuencas hidrográficas y los ecosistemas que regulan sus caudales; así como la generación de fuentes alternativas de electricidad.

Figura 4. Oferta hídrica per cápita en Colombia

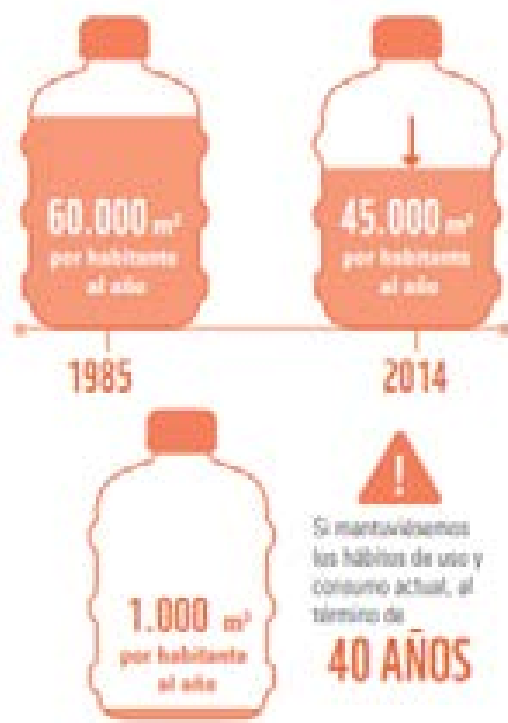

Fuente: WWF-Colombia 2017, Colombia Viva: Un país megadiverso de cara al futuro. Informe 2017. 
Desde esta perspectiva, el país debe hacer frente a los desafíos que impone el deterioro ambiental. Entre las múltiples acciones a desarrollar está incrementar las energías renovables no convencionales, además de mejorar la eficiencia energética. Una de las fuentes de energía renovable es la energía solar, la cual se obtiene a partir del aprovechamiento de la radiación electromagnética procedente del sol. Precisamente por ser una energía limpia, el Solar Decathlon (SD) le apuesta al uso de paneles fotovoltaicos para la generación de energía y la funcionalidad de la casa.

Un panel fotovoltaico consiste en una "asociación de células, encapsulada en dos capas de EVA (etileno-vinilo-acetato), entre una lámina frontal de vidrio y una capa posterior de un polímero termoplástico (normalmente tedlar). Este conjunto es enmarcado en una estructura de aluminio con el objetivo de aumentar la resistencia mecánica del conjunto y facilitar el anclaje del módulo a las estructuras de soporte" (Perpiñán, 2012, p. 25).

La UPME (2019) expresa que en Colombia existen registradas alrededor de 38 empresas dedicadas a la producción de energía solar o dispositivos tecnológicos generadores o funcionales con energía solar fotovoltaica principalmente. Según esta misma entidad, se estima que para antes del 2030 cerca del $10 \%$ del consumo energético en Colombia va a provenir de proyectos fotovoltaicos o solares. De este grupo de empresas se destaca Celsia S.A. E.S.P., como la principal productora de energía solar fotovoltaica del país, la cual se encuentra ubicada en Yumbo.

La suma de las razones expuestas sustenta la pertinencia del desarrollo de alternativas sostenibles con el medio ambiente, no solo desde la incorporación de fuentes de energía sostenibles, sino en la apropiación social de los cambios de paradigmas en diferentes dinámicas de lo humano, que exige el desgaste medio ambiental del país, y del mundo en general. 


\section{Dinámica del sector de la construcción en Colombia}

El Consejo Colombiano de Construcción Sostenible - CCCS - (2008), define la construcción sostenible como aquella que está en armonía con el sitio, hace uso de energía, agua y materiales de un modo eficiente y provee confort y salud a sus usuarios; es decir, la construcción sostenible abarca el espacio recreado por las edificaciones, el entorno y la manera cómo se integran para formar las ciudades.

Desde esta perspectiva, las construcciones sostenibles son un elemento del desarrollo urbano sostenible, el cual busca generar hábitats que no atenten contra el medio ambiente, proporcionando recursos urbanísticos suficientes, desde el diseño, la eficiencia energética y del agua, y la funcionalidad del espacio habitado.

Según Rodríguez Potes et al. (2018; p. 23), "los principios básicos del urbanismo sustentable están condensados en la Carta del Nuevo Urbanismo (CNU, 2001), los cuales se pueden aplicar en las diversas escalas de ordenación: edificio, barrio, bloque urbano, ciudad, región y país". Las construcciones sostenibles que se enmarcan en la denominada arquitectura sustentable, deben cumplir con los siguientes aspectos:

a. Reducir los gastos de los recursos empleados.

b. Reducir la contaminación del suelo, el aire y el agua.

c. Mejorar el confort interno y externo del edificio, preferentemente de manera pasiva.

d. Ahorrar recursos económicos y financieros en el proceso constructivo.

e. Reducir los desperdicios derivados del ciclo del inmueble (diseño, construcción, uso, mantenimiento y demolición).

f. Mejorar la tecnología que provee servicios a los inmuebles (aparatos, máquinas, mecánica y eléctricas)" (Rodríguez Potes et al., 2018, p. 25). 
La Agencia de los Estados Unidos para el Desarrollo Internacional (siglas en inglés USAID) (2018), indica que la construcción y ejecución de obras civiles o urbanísticas generan un impacto significativo sobre el medio ambiente y el cambio climático; agotando el $12 \%$ del agua potable global, el $38 \%$ de las emisiones globales de gases de efecto invernadero, el 40 $\%$ del consumo energético anual y casi el $50 \%$ de los recursos naturales (USAID, 2018).

La industria nacional de la construcción consume el $40 \%$ de la energía y el $60 \%$ de los materiales extraídos de la tierra, genera el $30 \%$ del $\mathrm{CO}_{2}$ y el $40 \%$ de los residuos (Ministerio de Medio Ambiente y Desarrollo Sostenible, 2018).

En cuanto a la dinámica de crecimiento del sector construcción en Colombia, según datos de la Cámara Colombiana de la Construcción - CAMACOL -, la tasa de crecimiento del sector de la construcción tuvo una contracción del 2,5\% en el 2018; aunque ha tenido una dinámica positiva en la última década (CAMACOL, 2018) (Ver Figura 5).

\section{Figura 5. Crecimiento (\%) del PIB y PIB construcción Colombia, 2001-2018*}

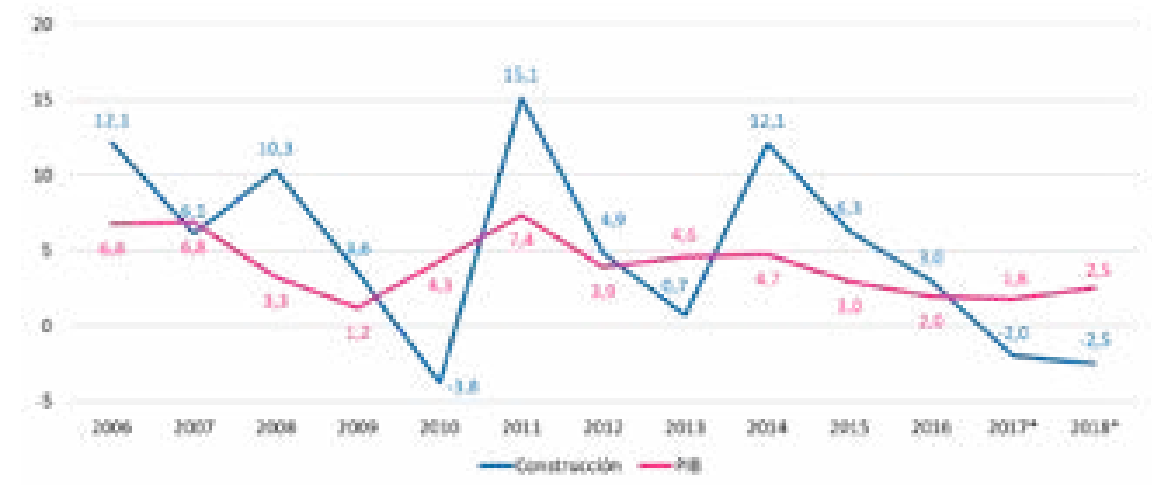

*Preliminar. Dato de 2018 acumulado a III trimestre Fuente: DANE - Cálculos Cámara de Comercio de Cali

La dinámica del crecimiento del sector construcción en los últimos tres años, se logra explicar a partir de las ventas de vivienda nueva en los principales departamentos del país. 


\section{Figura 6. Ventas de vivienda nueva principales departamentos (unidades) anual, 2016-2018.}

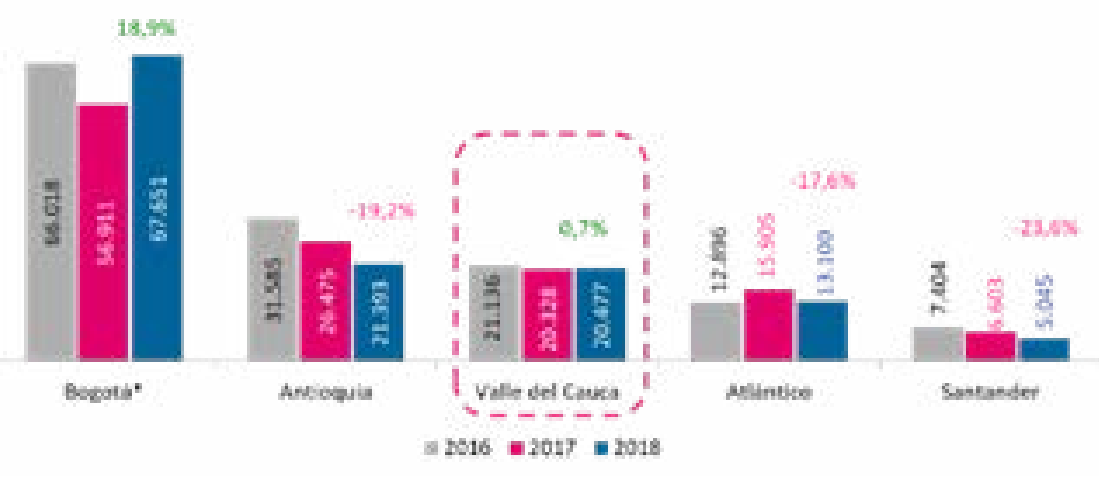

*incluye Cundinamarca

Fuente: Coordenada Urbana - Elaboración Cámara de Comercio de Cali

Vemos que Antioquia, Atlántico y Santander tuvieron una caída significativa en las ventas de vivienda nueva, mientras que Cundinamarca (incluye Bogotá) y el Valle del Cauca desarrollaron incrementos en dicho segmento. Sólo en el Valle del Cauca se vendieron 20477 unidades de vivienda en 2018, lo que representó un incremento del 0,7\% frente a 2017.

Tabla 1. Unidades de vivienda nueva vendidas por área de conurbación de Cali (ACC), febrero 2017-2019

\begin{tabular}{|c|c|c|c|}
\hline Municipio & $\mathbf{2 0 1 7}$ & $\mathbf{2 0 1 8}$ & $\mathbf{2 0 1 9}$ \\
\hline Cali & 644 & 724 & 542 \\
\hline Jamundí & 452 & 226 & 275 \\
\hline Candelaria & 136 & 338 & 271 \\
\hline Palmira & 192 & 159 & 85 \\
\hline Yumbo & 158 & 92 & 47 \\
\hline Puerto Tejada & 4 & 16 & 23 \\
\hline Total ACC & 1586 & 1555 & 1243 \\
\hline
\end{tabular}

Fuente: Coordenada Urbana - Elaboración Cámara de Comercio de Cali 
En el área de conurbación de Cali (ACC) se vendieron 1 243 unidades de viviendas nuevas en febrero de 2019; 56,4\% se vendieron en los municipios vecinos a Cali. Esto evidencia como el ACC genera procesos de densificación poblacional que desarrollan problemáticas de movilidad, aglomeración urbana y alto impacto ambiental.

Figura 7. Participación (\%) de la población del ACC en el Valle del Cauca, 1985-2020.

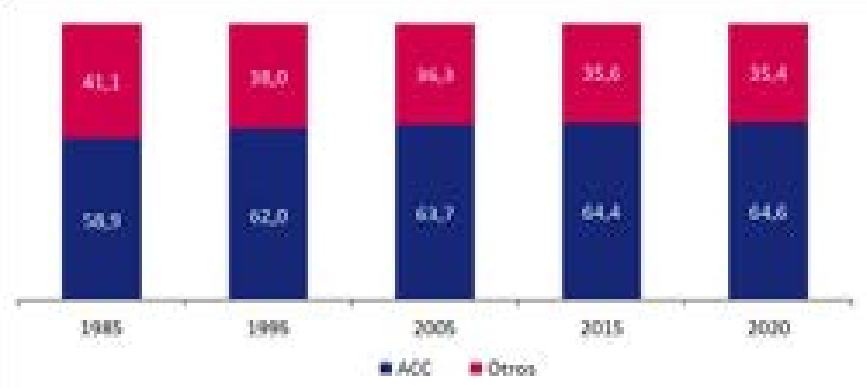

Fuente: DANE - Elaboración Cámara de Comercio de Cali

La población ubicada en el ACC pasaría de ser 58,9\% de la población del Valle del Cauca en 1985, a representar 64,6 \% en 2020 según proyecciones del DANE.

Figura 8. Distribución de las viviendas según tipo, $2018^{\text {pr }}$

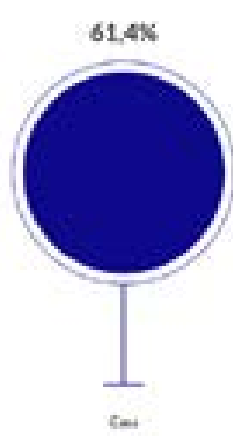

Fuente: DANE, Censos y Demografía, 2018. 
Según el DANE (2018), las viviendas tipo casa $(61,4 \%)$ y apartamento $(33,1 \%)$, son las formas habitacionales que lideran en Colombia. Por lo tanto, es oportuno diseñar espacios para el desarrollo de innovaciones de construcción sostenible que involucren los formatos de casa y apartamento.

Figura 9. Distribución (\%) de viviendas según acceso a servicios públicos domiciliarios en Colombia, $2018^{\mathrm{pr}}$

Energia elletaica

Aeuedacto

Aleartariliado

Cas natural

Recolecoion de basurac

Interret thoo movil

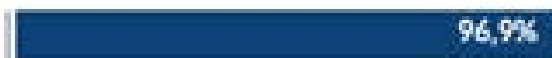

$86,6 \%$

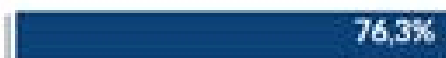

$67,1 \%$

$B 1,4 \%$

\section{8}

Fuente: DANE, Censos y Demografía, 2018.

Sobre la cobertura de servicios públicos, el 96,9\% de las viviendas en Colombia contaron con energía eléctrica en 2018, lo cual define una amplia cobertura energética en el país. Sin embargo, Colombia aún tiene importantes retos frente a la diversificación de la oferta, principalmente en energías limpias que permitan reducir el impacto ambiental en la generación y en el consumo energético de los hogares y el sector empresarial. 
Figura 10. Hogares y distribución por clase, 2018

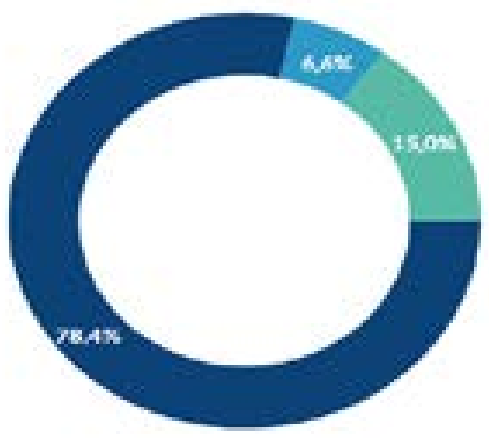

Fuente: DANE, Censos y Demografía, 2018.

El $85 \%$ de los hogares (viviendas) en Colombia se encuentran en espacios de aglomeración urbana. El 78,4 \% está en las cabeceras municipales y el 6,6 \% en centros poblados ${ }^{3}$. Esto expresa la necesidad de crear hábitats sostenibles con el desafío de dinámicas urbanas con alta densidad poblacional. En este sentido, la construcción sostenible no se limita al espacio de la vivienda, sino a la articulación de este microespacio habitacional, con el espacio urbano de interacción social.

3 Se define como una concentración de mínimo veinte (20) viviendas contiguas, vecinas o adosadas entre sí, ubicada en el área rural de un municipio o de un corregimiento departamental (DANE, 2019). 
Figura 11. Hogares particulares según número de personas, 2005 - 2018

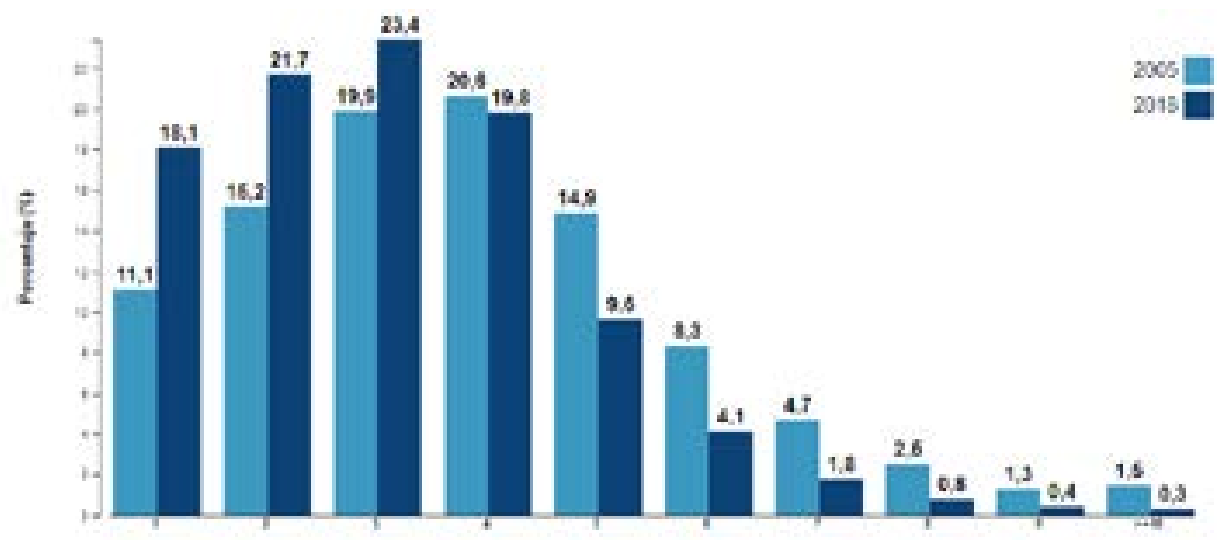

Fuente: DANE, Censos y Demografía, 2018.

El tamaño de los hogares más representativo en Colombia en el 2005 fue de 4 personas, alcanzando el 20,6\% del total de hogares. Sin embargo, en el 2018 la composición por hogar más representativa se redujo a 3 personas, con un 23,4 \%. En ese mismo periodo se presentó un cambio significativo en el número de hogares unipersonales y de 2 personas; mientras en el 2005 los hogares unipersonales representaban el 11,1\% del total nacional, en el 2018 representaron el 18,1 \%. A su vez, los hogares de 2 personas eran el $15,2 \%$ en el 2005, y pasaron a 21,7 \% en 2018 (DANE, 2018). El menor número de personas por hogar induce una mayor aglomeración espacial, ya que menos personas ocupan el mismo espacio (vivienda); pero más viviendas ocupan el espacio urbano.

Estos cambios en la composición familiar implican nuevos desafíos en diversos frentes; en particular representan una señal de cambio que debe ser recogida por los actores relacionados con la industria de la construcción y el diseño de política nacional de vivienda y desarrollo de territorio urbano.

Según el CCCS (2018), en Colombia existe un mercado dinámico con edificaciones que aportan a mitigar el cambio climático, validados por estándares internacionales como LEED (Estados Unidos), y el nuevo sistema de certificación CASA Colombia. LEED (por sus siglas en inglés, Leadership in Energy \& Environmental Design) es el sistema de certificación más utilizado 
en el mundo para el diseño, construcción, mantenimiento y operación de construcciones sostenibles. Al utilizar menos energía, los espacios certificados LEED ahorran dinero a las familias y empresas, reducen las emisiones de carbono y contribuyen con ambientes saludables para el uso de residentes, trabajadores y la comunidad en general. Las categorías de evaluación son:
a. Proceso integrativo de diseño
b. Sitios sostenibles
c. Uso eficiente del agua
d. Energía y atmósfera
e. Calidad ambiental interior
f. Materiales y recursos
g. Innovación
h. Prioridad regional

A diciembre de 2018, 151 proyectos inmobiliarios en Colombia contaban con la certificación LEED, con un espacio habitacional de 2,3 millones de $\mathrm{m}^{2} ;$ y 223 proyectos se encontraban en trámite de certificación LEED, con un total de 3,9 millones de $\mathrm{m} 2$ de espacio habitacional (CCCS, 2018).

Por otro lado, la Guía de Construcción Sostenible para el Ahorro de Agua y de Energía en Edificaciones, fue elaborada por el Ministerio de Vivienda, Ciudad y Territorio, la Corporación Financiera Internacional (IFC) del Banco Mundial y la Cámara Colombiana de la Construcción (CAMACOL), con el objetivo de establecer los estándares de ahorro de agua y energía de mínimo un $15 \%$ para edificaciones nuevas.

Desde esta perspectiva, el diseño bioclimático de la vivienda y la correcta orientación solar permite regular los cambios climáticos y de temperatura, conservando un perfecto confort térmico y ambiental sin gastos energéticos adicionales, a la vez que se mantiene una correcta renovación del aire, respetando la respiración del edificio por todos sus poros (paredes y techo) y evitando los aislamientos de poro cerrado y las pinturas plastificantes. Según CCCS (2018), los proyectos inmobiliarios con uso no residencial pueden tener un eventual sobrecosto de hasta $4 \%$, con un periodo muy competitivo de retorno de la inversión de entre 1 y 3 años, por la curva de aprendizaje desarrollada en los últimos años. 
La importancia que ha tenido el sector de la construcción en la última década en Colombia, así como los nuevos desafíos que implica la composición de los hogares en Colombia y la densificación poblacional en los espacios urbanos, implican nuevos desarrollos de construcción sostenible que limitan el impacto ambiental que genera la construcción y la funcionalidad de una vivienda. Uno de los principales aportes para dicha sostenibilidad ambiental es la incorporación en las edificaciones, de todo tipo y fin, la energía solar, como fuente de energía para la funcionalidad integral de la vivienda.

Figura 12. Distribución (\%) de la matriz de generación de energía en Colombia según fuente 1998 y 2018
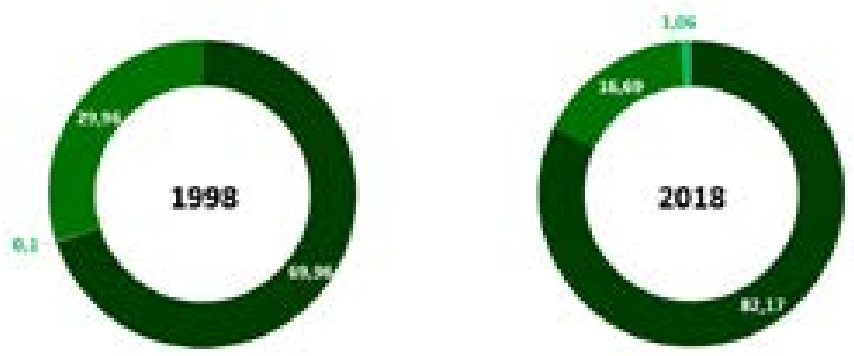

Otros: Solar y Energía Eólica

Fuente: XM - Cálculos Cámara de Comercio de Cali

La generación de energía en 2018 fue 68.942.970 MW/h. La participación de la cogeneración en la matriz energética pasó de $0,1 \%$ en 1998 a $1,1 \%$ en 2018. Lo anterior evidencia que en el país se vienen desarrollando alternativas para diversificar la oferta energética, aunque siguen siendo insuficientes; siendo la energía solar una de las fuentes de generación de energía más renovables y con menor impacto ambiental. 


\section{Figura 13. Celsia Solar Yumbo}

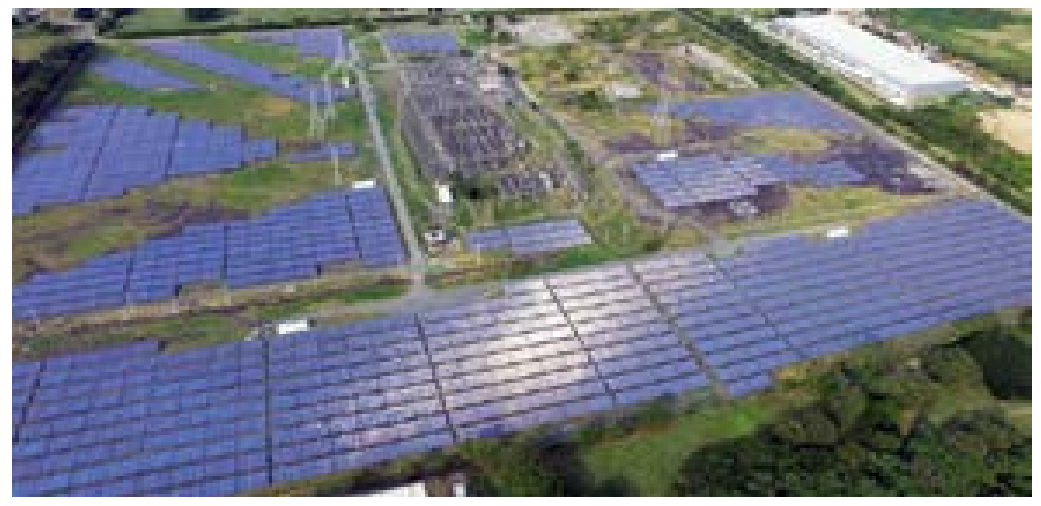

Fuente: EPSA - CELSIA, 2018; Tomado de www.celsia.com

El proyecto Celsia Solar Yumbo, la granja solar más extensa del país que cuenta con 35000 paneles solares en un terreno de 18 hectáreas, la cual genera $16 \mathrm{GWh}$ al año aproximadamente, equivalente al consumo básico mensual de energía de 8000 viviendas, es el primer megaproyecto desarrollado en el país de energía solar. El proyecto permite evitar la emisión de unas 6 600 toneladas de $\mathrm{CO}_{2}$ al año.

Desde esta perspectiva es imperativo desarrollar viviendas (diseños y prototipos), con factibilidad técnica y comercial, sostenibles a partir de energía solar; generando estrategias de apropiación de ese conocimiento especializado, en conocimiento práctico para la población en general, entendiendo la incorporación de energía solar en la funcionalidad de una vivienda, y la sostenibilidad ambiental como una responsabilidad social comunitaria.

\section{Estadísticas de población con movilidad reducida en Colombia y América Latina}

Según la Sala Situacional de las Personas con Discapacidad (PCD) del Ministerio de Salud y Protección Social (MSPS), el $3 \%$ de la población colombiana; es decir, 1379001 personas, tiene algún tipo de discapacidad. A través del Registro de Localización y Caracterización de Personas con Discapacidad (RLCPD) se realiza la medición y seguimiento a dicha población. 
Figura 14. Estructura piramidal de la población con discapacidad en Colombia, 2018.

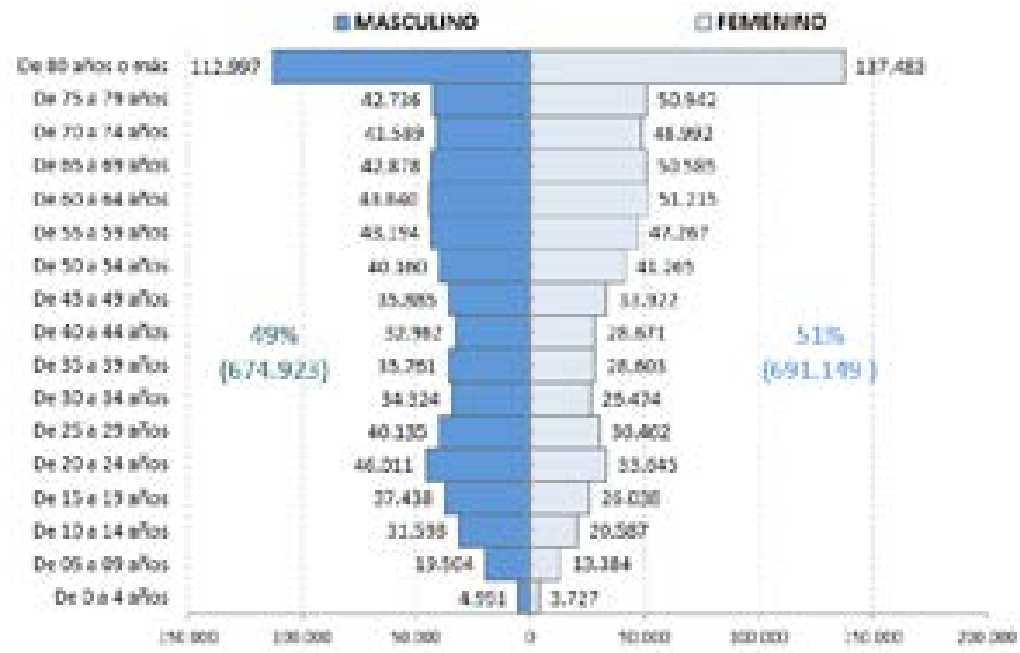

Fuente: MSPS: SISPRO, RLCPD, febrero 2018.

Respecto al sexo, no existe una diferencia considerable en la población con algún tipo de discapacidad; sin embargo, sí existe una brecha significativa respecto a la edad. El $58 \%$ de las personas con discapacidad registradas en el RLCPD (2018) son mayores de 50 años y el $33 \%$ de las personas mayores de 80 años tienen algún tipo de discapacidad (RLCPD, 2018), principalmente asociado a problemas de movilidad reducida, lo cual define grandes desafíos en la política pública sobre el asunto, incluidos los diseños funcionales de las viviendas, en virtud de que la edad se convierte en un factor de riesgo de discapacidad. 
Figura 15. Personas con discapacidad según pertenencia étnica, 2018.

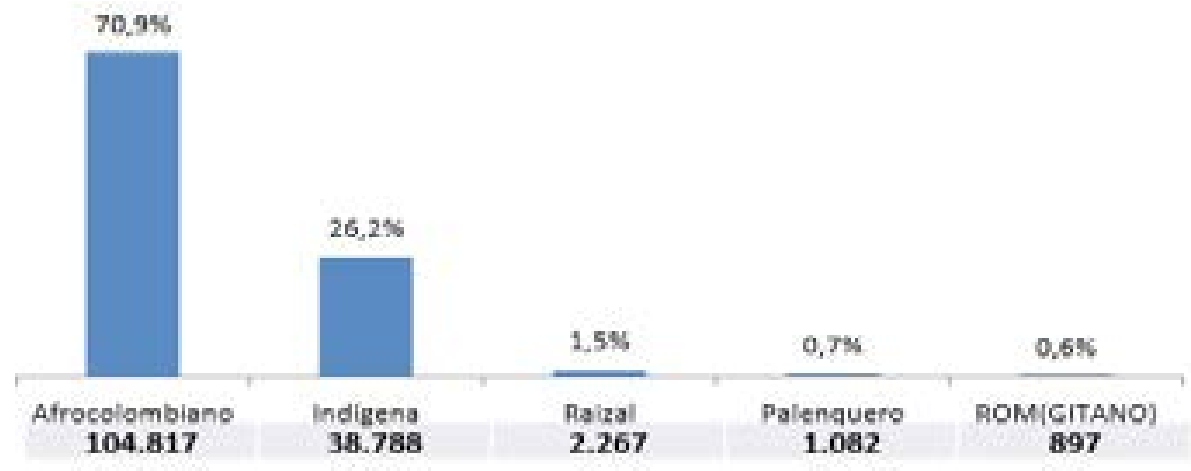

Fuente: MSPS: SISPRO, RLCPD, febrero 2018.

Un elemento que refleja amplias brechas respecto al desarrollo de algún tipo de discapacidad es la pertenencia a una etnia. El $70,9 \%$ de las personas con discapacidad que manifestaron pertenecer a una etnia, son afrocolombianos; seguidos por indígenas $(26,2 \%)$. Esto genera desafíos para departamentos como el Valle del Cauca, específicamente para Cali, que registra la mayor población afrocolombiana del país, con un total de 635805 afrocolombianos, siendo el $26 \%$ del total de población de la ciudad (DANE, 2018).

Según el MSPS (2018) las principales fuentes de algún tipo de discapacidad están asociadas a alguna enfermedad en general, accidentes viales, alteración genética hereditaria, conflicto armado, entre otras (RLCPD, 2018). 
Tabla 2. Personas con discapacidad y víctima del conflicto, según el tipo de alteración. RLCPD-RUV. 2017*

\begin{tabular}{|c|c|c|}
\hline Tipo de alteración & $\begin{array}{c}\text { Número de } \\
\text { personas }\end{array}$ & $\%$ \\
\hline El movimiento del cuerpo, manos, brazos, piernas & 67084 & $38 \%$ \\
\hline El sistema nervioso & 40707 & $23 \%$ \\
\hline Los ojos & 27664 & $16 \%$ \\
\hline El sistema cardiorrespiratorio y las defensas & 14595 & $8 \%$ \\
\hline La voz y el habla & 8715 & $5 \%$ \\
\hline Los oídos & 8691 & $5 \%$ \\
\hline La digestión, el metabolismo, las hormonas & 4534 & $3 \%$ \\
\hline El sistema genital y reproductivo & 2703 & $2 \%$ \\
\hline La piel & 1469 & $1 \%$ \\
\hline Los demás órganos de los sentidos (olfato, tacto y gusto) & 874 & $0 \%$ \\
\hline
\end{tabular}

*Una persona puede tener más de una alteración Fuente: RLCPD, 2017.

De las personas con discapacidad, 177742 se registran como víctimas en el Registro Único de Victimas (RUV); es decir, el $13 \%$ del total de personas con algún tipo de discapacidad son víctimas del conflicto armado (RLCPD, 2017). De esta población, el $38 \%$ tiene alterado el movimiento del cuerpo, manos, brazos y piernas; lo cual define la categoría de movilidad reducida.

\section{Figura 16. Personas con discapacidad según la alteración que más le afecte, 2018.}

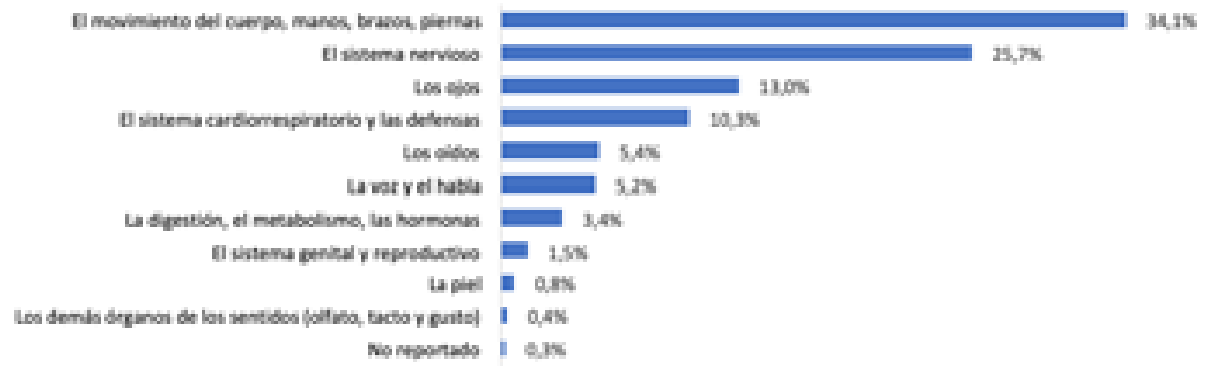

Fuente: MSPS: SISPRO, RLCPD, febrero 2018. 
El 34,1\% de la población total con algún tipo de discapacidad, (470 215 personas), expresaron que la alteración que más le afecta es el movimiento del cuerpo, mientras el 25,7 \%, (350 216 personas), refirió el sistema nervioso como consecuencia del conflicto armado (RLCPD, 2018). En general, el movimiento del cuerpo es la alteración que más afecta a la población con discapacidad, sin una diferencia significativa respecto al género.

\section{Tabla 3. Personas con discapacidad según tenencia de servicios públicos - RLCPD}

\begin{tabular}{|c|c|c|}
\hline Servicio público & No tiene & $\%$ \\
\hline Acueducto & 258595 & $18,8 \%$ \\
\hline Agua no potable & 349564 & $25,3 \%$ \\
\hline Energía eléctrica & 60849 & $4,4 \%$ \\
\hline Alcantarillado & 423165 & $30,7 \%$ \\
\hline Teléfono & 805800 & $58,4 \%$ \\
\hline Ningún servicio & 40907 & $3 \%$ \\
\hline
\end{tabular}

Fuente: MSPS: SISPRO, RLCPD, febrero 2018.

El $80 \%$ de las personas con algún tipo de discapacidad pertenecen a los estratos socioeconómicos 1 y 2 . Así mismo, el $64 \%$ no tienen ningún tipo de ingreso y el $21,6 \%$ reciben menos de $\$ 500000$ mensuales. El 30,7\% de las personas con algún tipo de discapacidad no cuentan con un sistema de alcantarillado, (RLCPD, 2018). Esto expresa un alto grado de vulnerabilidad socioeconómica de la población con movilidad reducida.

Figura 17. Personas con discapacidad según la persona quien le ayuda, 2018.

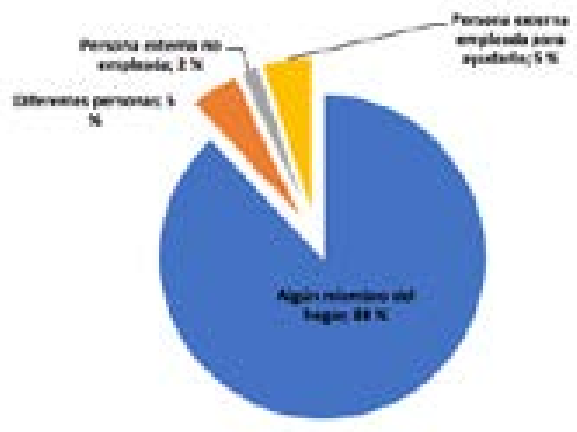

Fuente: MSPS: SISPRO, RLCPD, febrero 2018. 
El $40 \%$ de la población con algún tipo de discapacidad (559 266 personas) expresaron necesitar ayuda de otras personas para realizar sus actividades diarias. El $88 \%$ de esta población recibe ayuda de un miembro del hogar, y sólo el $5 \%$ de una persona (enfermera) contratada. Por otro lado, el $22 \%$ de la población total con algún tipo de discapacidad refirieron necesitar ayuda técnica (bastones, muletas, etc.). La vulnerabilidad frente a la autonomía que recrea algún tipo de discapacidad, principalmente la movilidad reducida, se complica cuando el $8,5 \%$ del total de la población con algún tipo de discapacidad refieren una actitud negativa por parte de los familiares, lo cual les impide el desarrollo de sus actividades diarias con mayor autonomía (RLCPD, 2018).

\section{A manera de conclusión}

De acuerdo con los referentes utilizados en este escrito, la dinámica de la economía de mercado, ha generado sensibilidad de manera obligatoria frente a los desafíos de los aspectos ambientales, estudios que miden en términos cualitativos y cuantitativos los impactos que genera la oferta de unidades habitacionales en el mundo; estos análisis han generado el uso de términos traídos de las disciplinas económico-administrativas como sobregiro, pero enfocado en la contabilidad de recursos que se utilizan en la industria de la construcción comparado con la capacidad del territorio para producir recursos renovables y asumir los residuos propios de la actividad.

Los resultados de este enfoque cuantitativo demuestran el estado crítico en las denominadas economías desarrolladas, en donde aparece Estados Unidos como el país con mayor desequilibrio en estos indicadores, seguido por los países de mayor desarrollo en los demás continentes como Australia en Oceanía, Alemania en Europa, Rusia en Euro-Asia y Corea del Sur en Asia. Estos hallazgos demuestran que la insostenibilidad ambiental del planeta es una realidad dado que la huella ecológica desborda la generación de recursos en estos territorios (biocapacidad), que en términos globales son de gran impacto en la contabilidad ambiental del globo. 
En el caso de América Latina, Brasil aparece como el país más crítico en términos de desbalance en estos indicadores y para el caso colombiano, según estudios de gremios relacionados con el sector, presenta un superávit por ahora de biocapacidad frente a la huella ecológica, pero tendrá que ser observada por los empresarios y por el Estado so pena de caer en déficit en indicadores en detrimento de la vida en la región; Estos aspectos se están considerando y normatizando en Colombia a través de la Carta de Urbanismo (CNU, 2001).

Los estudios relacionados con el sector en términos de sostenibilidad e inclusión, deberán estar alineados con los ODS de las Naciones Unidas, y específicamente con el ODS 11, el cual considera aspectos que trascienden los indicadores cuantitativos expuestos anteriormente en el entendido de que existen otras variables de orden cualitativo como la ubicación geográfica de las soluciones habitacionales para las poblaciones menos favorecidas de los territorios.

Si se desean lograr ciudades sostenibles en Colombia, el ODS 11, se debe focalizar en los efectos de la llamada segregación socioespacial, aparte de los enfoques tradicionales de atender el déficit de vivienda o los impactos ambientales de los desarrollos urbanísticos. El concepto de sostenibilidad debe ser abordado desde una óptica holística que considere las variables no solo de carencia sino de soluciones que involucren a los actores a la vida social integrada de las ciudades, a través del cierre de la brecha de la segregación socioespacial.

Dicho de otro modo, la problemática no se reduce solamente a proponer políticas públicas y proyectos consecuentes con estas, sino a evaluar que las soluciones de sostenibilidad no necesariamente se resuelven con la oferta de una solución habitacional, ni la inclusión se resuelve solamente con los accesos adecuados para personas con discapacidades, sino que trascienden estos conceptos si se hace una mirada amplia del problema. El ODS 11 postula "lograr que las ciudades y los asentamientos humanos sean inclusivos, seguros, resilientes y sostenibles" (Organización de las Naciones Unidas [ONU], s. f.). 
Si se aborda este objetivo con toda su carga de impacto en la sociedad, entonces se tendrá que entender que las soluciones de desarrollo urbanístico tendrán que afrontar la inclusión como la posibilidad de accesos a las unidades propuestas y la sostenibilidad desde todos los ámbitos (social, económico, ambiental) en entornos de ciudad que incluyan a las poblaciones en los centros de desarrollo urbano y no de manera excluyente en las periferias de la ciudad que de hecho implicaría un problema de segregación socioespacial.

Desde esta perspectiva, el denominado "efecto vecindario", que implica que, para franjas de la población con menos recursos, habiten en sectores (barrios, comunas) totalmente pobres, impacta negativamente las posibilidades de estos actores sociales, sus aspiraciones de inclusión y movilidad social comparado con los pobladores que se han establecido en barrios de configuración más heterogénea (Kaztman, 2007; Sampson, 2012; Wilson, 1996). De este tamaño es el desafío del sector y la política pública.

\section{Bibliografía}

Arias, Fabio (2006). Desarrollo sostenible y sus indicadores. Revista Sociedad y Economía, N. ${ }^{\circ} 11$, julio-diciembre 2006, pp. 200-2029, ISSN 1657-6357. Universidad del Valle, Colombia.

Callon, Michel (1994). Is Science a Public Good? Science, Technology and Human Values 19(4): 395-424.

CAMACOL. (2018). Informe de actividad edificadora. Recuperado de http://camacolatlantico.org/wp-content/uploads/bsk-pdf-manager/

CELSIA (2018). Celsia Solar Yumbo. Recuperado de https://www.celsia.com/es/sala-prensa/con-celsia-solar-yumbo-colombia-cumple-un-a241o-generando-energ237a-renovable-a-gran-escala

CNU. (2001). The Charter of the New Urbanism. Recuperado de https://www.cnu.org/ charter

Consejo Colombiano de Construcción Sostenible (2016). Avances en construcción sostenible en Colombia. Bogotá: CCCS.

Consejo Colombiano de Construcción Sostenible (2018). Avances en construcción sostenible en Colombia. Bogotá: CCCS.

DANE. (2018). Censo Poblacional 2018. Recuperado de http://www.dane.gov.co

Kaztman, R. (2007). La calidad de las relaciones sociales en las grandes ciudades de América Latina: viejos y nuevos determinantes. Pensamiento Iberoamericano, 1, 177-205. Recuperado de https://dialnet.unirioja.es/servlet/articulo?codigo $=2873233$

Ministerio de Ambiente y Desarrollo Sostenible (2018). Minambiente reglamenta manejo y disposición de residuos de construcción y escombros. Recuperado de http:// www.minambiente.gov.co/index.php/noticias/2681-minambiente-reglamentamanejo-y-disposicion-de-residuos-de-construcciony-escombros

Ministerio de Salud y Protección Social (2018). Sala Situacional de las personas con discapacidad (PCD) - 2018. Consultado en: https://www.minsalud.gov.co/sites/rid/ Lists/BibliotecaDigital/RIDE/DE/PS/sala-situacional-discapacidad-junio-2018.pdf 
Nowotny, Helga, Peter Scott and Michael Gibbons (2003). Intoduction, Mode 2 Revisted: The New Production of Knowledge. Minerva, 41: 179-194.

Perpiñán Lamigueiro, Oscar (2012). Energía Solar Fotovoltaica. Creative Commons, p. 25.

Organización Mundial de la Salud, OMS (2001). Clasificación internacional del funcionamiento, de la discapacidad y de la salud - CIF. OMS, Ginebra, 2001.

Organización Mundial de la Salud, OMS (2017). Informe Mundial sobre la Discapacidad. OMS, Ginebra, 2017.

Pabón, R. (2018). Apropiación social del conocimiento: una aproximación teórica y perspectivas para Colombia. Revista Educación y Humanismo, 20(34), 116-139.

Rodríguez Potes, L., Osorio Chávez, H., Villadiego Bernal, K., \& Padilla-Llano, S. (2018). Arquitectura y urbanismo sostenible en Colombia. Una mirada al marco reglamentario. Bitácora Urbano Territorial, 28(3), 19-26.

USAID. (2013). Documento técnico de soporte. Recomendaciones de Política Pública de Construcción y Urbanismo Sostenible. Informe final. Bogotá: USAID.

Vessuri, Hebe (1999). National Social Science Systems in Latin America. En: A.Kazancigil y D.Makinson (Eds) World Social Science Report. Pp.109-121, París: UNESCO.

Wackernagel, M., Onisto, L., Bello, P., Callejas, A., López, I. S., Méndez, J. Suárez Guerrero, A. I., Suárez Guerrero, M. G. (1999). National natural capital accounting with the ecological footprint concept, Ecological Economics, N.o 29, pp.375-390.

World Commission on Environment and Development (1987). Our Common Future, Oxford, Oxford University Press.

WWF-Colombia (2017). Colombia Viva: un país megadiverso de cara al futuro. Informe 2017. Cali: WWF-Colombia. 